\title{
A simple but accurate device and method for bending and stress measurement of metallic structures
}

\author{
Cosmin Mihai Miriţoiu ${ }^{1}$ \\ ${ }^{\text {I }}$ Faculty of Mechanics, University of Craiova, Vehicles, Transports and Industrial Engineering Department, \\ Calea Bucureşti street, no. 107, Craiova, code 200512, Romania)
}

\begin{abstract}
In this paper I present a device and method for bending a metallic structure. This device has a simple construction and can be applied for bending to all metallic structures that have two longitudinal beams connected by transversal beams with multiple supporting columns. I have determined the stress in one longitudinal beam by using strain gauge measurement. The strain gauge measurement is a technique widely used in experimental mechanics for more than 50 years. I have compared the obtained experimental results with the ones acquired from an analytical model that I presented before in a scientific paper. The errors between the two methods (experimental and analytical) are very small, under $4 \%$.
\end{abstract}

Keywords - metallic structure, device, bending, strain gauge, stress

\section{INTRODUCTION}

The classical bending method of standardized specimens consists in using testing machines equipped with proper devices, like the ones presented in [Rosca et al., 2007] [1]. The most classical device for bending consists of two supporting rollers and another one used to apply the force. There are also other many devices and methods for bending structures, especially described in patents. For example, in [Codatto, 2006] [2] it is presented a method and a bending device for a portion of an element in a bending machine comprising a bending assembly, a supporting plane and an electronic processing unit, wherein the bending process is filmed and displayed on a screen. A bending method and device for a bending machine for oblong metal elements is described in [Del Fabro, 2009][3]. This device comprises a plurality of bending units provided with contrast pins, a bending support that defines a bending plane on which the specimens are positioned, and bending pins mounted mobile around the contrast pins, so as to bend the oblong metal elements, in cooperation with the contrast pins. A device for bending plates is shown in [Ernst and Manfred, 2002][4]. The edge zone of the sheet that will be folded is clamped between the upper and lower dies and a shaping edge bends the plate along the folding line. The shaping edge swings on a rotating body in a housing, so that the straight closing movement of the press is converted into a swing movement at the folding edge. The movement of the lower die is also converted into a swing motion, to swing the shaping edge. In [Fischreder and Reiter, 2012] [5] it is described a bending press, a production device and a method for operating such a press, comprising a workpiece handling device, a central control device, a workpiece positioning device with a first arresting device and with at least one further arresting device with arresting fingers wich are adjustable in an $\mathrm{X}$ axial position extending perpendicularly in relation to a bending plane for positioning a workpiece on a first bending tool for a bending operation between the first bending tool and a second bending tool that is adjustable in relation to said first bending tool. A bending device that bends a workpiece is presented in [Yogo, 2012] [6]. The device is provided with a bending mechanism that bends a workpiece, a fixing table on which a chuck mechanism that grips the workpiece is mounted, an articulated robot to which the bending mechanism is attached, and a control unit that controls the operations of the bending mechanism, the chuck mechanism and the articulated robot. In [Shigeki et al., 2011] [7] it is solved the problem of executing the heat treatment of a metal tube even in a portion in which an in-tube insertion and diameter expansion mechanism is inserted in a hollow part of the metal tube to be bent. In [Yoshiaki, 2011] [8] is is solved the problem of work efficiency improvement by maintaining eccentric amount of a bar material in constant during bending and simplifying a structure of a previously used device for this kind of problem. Both patents contain devices and methods for bending metal tubes and metal bar material. Some inverstigations regarding the determination of a bending angle for a rotor blade from a wind turbine system were made in [Felix et al., 2012] [9], where it is detailed a method and device.

The main contribution made in this paper is a device, with classical components, but assembled in an original form and used to bend metallic structures with several supporting columns. Also, in order to determine the stress from a longitudinal beam I have used the strain gauge measurements. This method has been used for the measurement of strain (and stress) for more than 50 years. In order to obtain proper experimental results, we 
have taken into account the recommendations presented in [Montero et al., 2011] [10]. This technique is used in many scientifical papers. For example, in [Ajovalaist and Zucarello, 2005] [11] the local reinforcement effect in tension is studied by using a simple theoretical model by considering a strain gauge mounted on a semi-infinite plate having the same width of the strain gauge and subjected to a uniaxial tension load. Neglecting the effect of the adhesive layer and considering the interface shear stress as an exponential distribution, the proposed model gives a closed-form solution. Strain gauge technique can also be applied for residual stress determination. For example, in [Litos et al., 2005] [12] it is made a simulation of strain gauge thermal effects during residual stress hole drilling measurements. The paper is divided in two major parts: the first part is devoted to the evaluation of heat flux from the drilling tool to the drilled material using the sample surface temperature measured by thermography and the second part deals with determination of real strain and strain gauge thermal output (apparent strain) at the strain gauge location during and after drilling. There are presented comparisons of simulated and experimentally determined temperatures and strains. It is described a destructive, sectioning method for measuring residual stress in welded panels, using electric strain gauges. The sectioning method is verified by a numerical determination of residual stress in welded panels. The results have shown that by using reasonably priced equipment there are obtained rapidly and accurate results to estimate the residual stress in a welded structure. Avalle and Goglio [1997] [13] have made a research regarding the static lateral compression of aluminium tubes using strain gauge measurements and theoretical models. The aim of the work was to obtain an experimental insight regarding the strain field during lateral compression and to verify the known theoretical models. The experimental activity involved aluminium tubes of 80 and $100 \mathrm{~mm}$ diameter and $2 \mathrm{~mm}$ wall thickness. Up to thirty strain gauges were applied on the internal and external surfaces of each tube. Many papers also present the stress-strain state that occurs in the wheel-railtrack assembly. For example, in [Faur et al., 2007] [14] it is made an analysis on this assembly in two methods: using the finite element analysis and the strain gauge measurements on the railtrack in connection with various run speed values of the tram. Jonsson et $a l$, [1997] [15] has made a measurement of wheel-rail interaction forces using strain gauge technique. The experimental results are compared with the ones obtained from finite element modelling. In [Bandula-Heva and Dhanasekar, 2011] [16] it is used the strain gauge method and Image Analysis to determine the stress-strain characteristics of railhead steel. Coupons obtained at varying depths of the railhead were tested under axial tension and the strains were measured using strain gauges and an image analysis technique, known as Particle Image Velocimetry.

\section{THE EXPERIMENTAL DEVICE AND RESULTS}

The metallic structure that is subjected to bending is presented in fig. 1, comprising two longitudinal beams marked with LB, three transversal beams marked with TB and six supporting columns marked with SC. I have marked with $\mathrm{F}$ and $\mathrm{P}$ the areas where the forces will be applied.

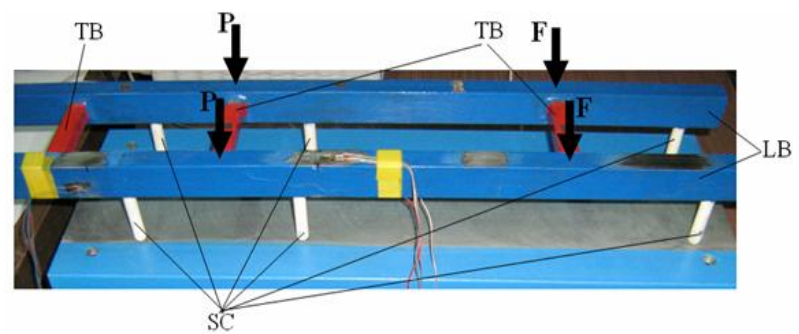

Fig.1. The studied metallic structure

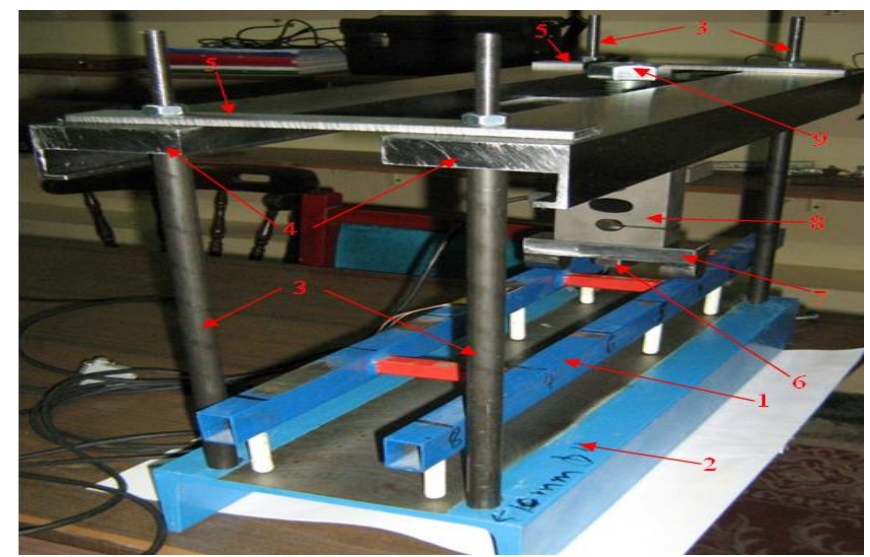

Fig. 2. The device used to bend parts of the studied metallic structure. General view 
A simple but accurate device and method for bending and stress measurement of metallic structures
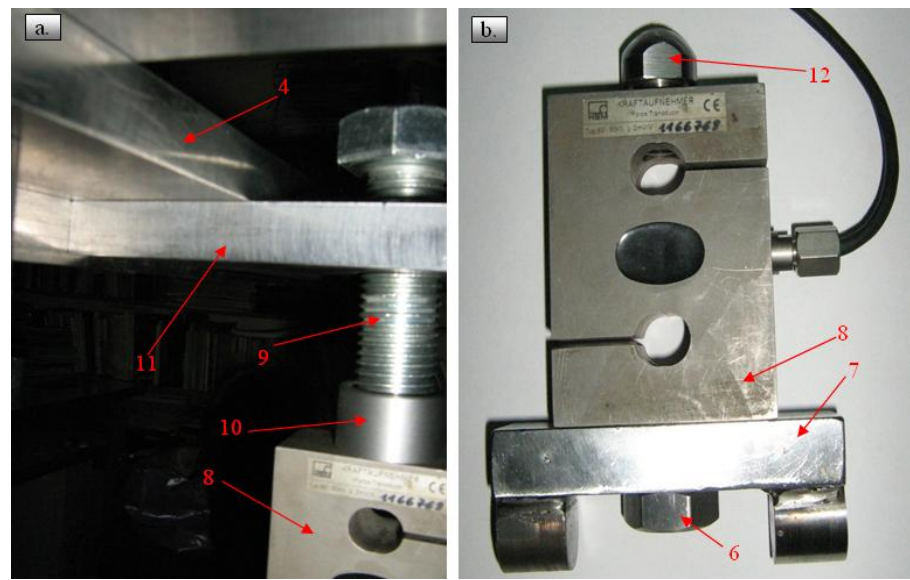

Fig.3. Detail in the area of the sliding plate 11 ; b. Detail with the loading assembled structure

From fig. 2 and 3 we can see the next parts of the experimental device: 1 - the analyzed metallic structure; 2- massive support U profile; 3 - four pieces of supporting columns; 4- two pieces of upper plates that guide the sliding plate $10 ; 5$ - two pieces of transversal plates for stiffening; 6 - M20 screw for fixing and centering the force transducer; 7 - the piece used for loading the structure; 8 -force transducer $\mathrm{S} 9$ type produced by HBM used to measure the force that loads the structure; 9- M30 screw used to apply the clamping force; 10small pipe used for guiding and centering the screw 9; 12- M20 screw used to take the force applied by the screw 9 and transmit it to the force transducer.

To determine the stresses in the longitudinal beams, I have mounted four strain gauges, in a half bridge connection on the structure. These are presented in fig. 4 and 5. The measuring system is SPIDER 8, produced by HBM presented in fig. 6 .

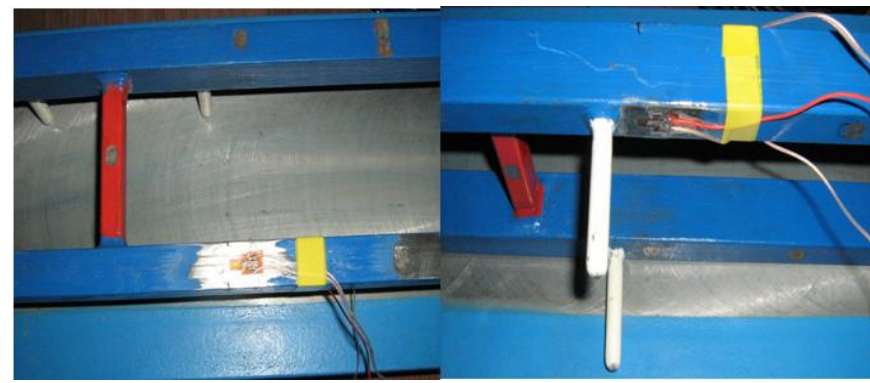

Fig. 4. The first half bridge

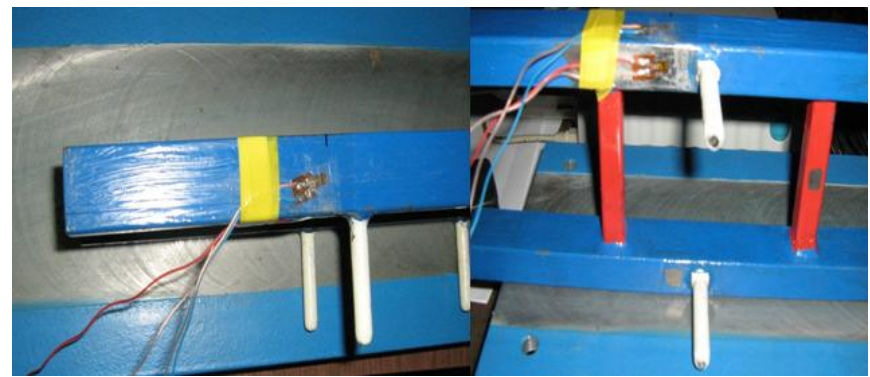

Fig. 5. The second half bridge

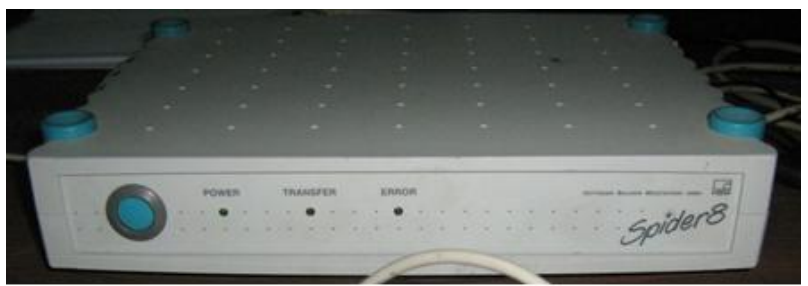

Fig. 6. The data acquisition system SPIDER 8 
A simple but accurate device and method for bending and stress measurement of metallic structures

I have loaded the structure in two variants: $\mathrm{P}=7,0155 \mathrm{kN}$ (variant 1) and $\mathrm{F}=5,0756 \mathrm{kN}$ (variant 2). $\mathrm{By}$ processing the experimental results, I have computed the von Mises stress in MPa values. To do this, I have created an own software to directly determine the von Mises stress in the Test Point programming software. I have marked with $\sigma_{1 \exp }$ the stress obtained with the first half bridge and with $\sigma_{2 \exp }$ the stress obtained with the second half bridge.

In fig. 7 I have shown the next parameters obtained experimentally: the force distribution (measured by the force transducer) with black line and the stress distribution with red (for the first half bridge) and blue (for the second half bridge) lines (measured by the strain gauges) for the variant 1 loading case. We have also marked with letters these parameters. In fig. 8 it is drawn the $\sigma_{1 \exp }$ variation depending on the value of force P. In fig. 9, I have shown the same parameters like in fig. 7, but for the second loading variant.

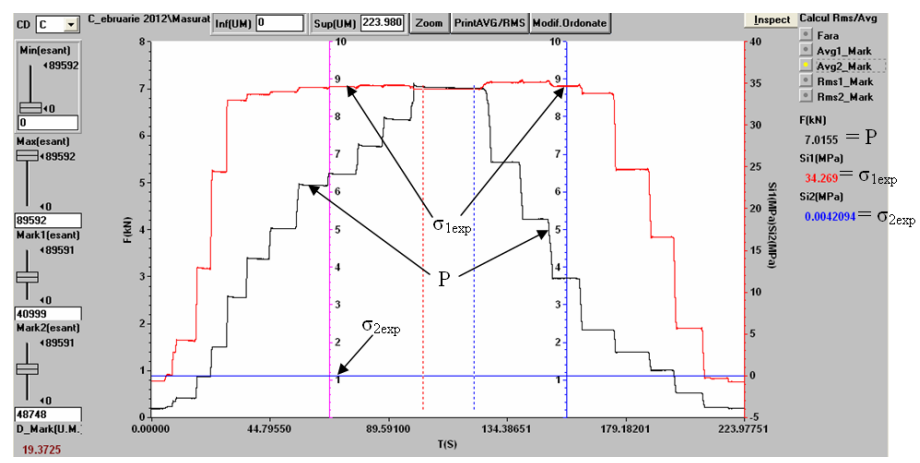

Fig. 7. The stress and force distributions in variant 1

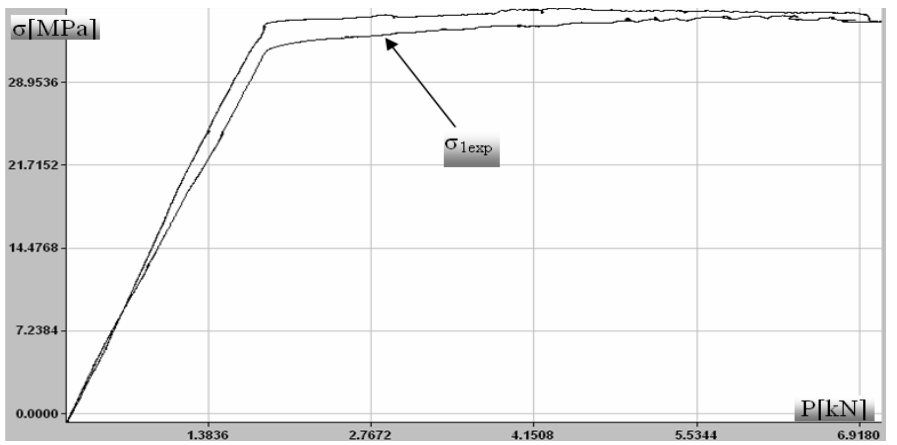

Fig. 8. The $\sigma_{1 \exp }$ variation depending on the value of force $\mathrm{P}$ in variant 1

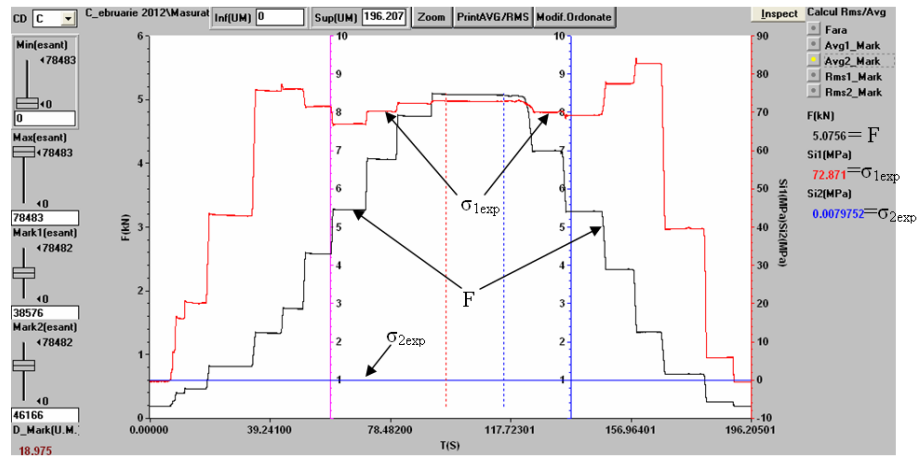

Fig. 9. The stress and force distributions in variant 2

\section{CONCLUSIONS}

In this paper we have presented a device and method for bending and stress measurement of metallic structures. The device has a very simple structure, different from the ones existing in the engineering literature, and can be used for bending metallic structures with two longitudinal beams connected by many transversal beams and having multiple supporting columns. Although the device has common parts, their assembly is original.

To determine the stresses from the bending loading, we have bonded four strain gauges on the structure. Most of the data acquisition software programs furnish strain results from the stress gauge measurement. That is why we

ISSN: 2250-3021 
A simple but accurate device and method for bending and stress measurement of metallic structures have created an own software to directly determine the von Mises stress in the Test Point programming software. This software can be applied to any structure for von Mises stress determining using experimental results from strain gauge measurements. We have considered two loading variants and we have experimentally obtained the force and the von Mises stress distributions in fig. 7, 8 and 9. From these figures, we can extract the following conclusions:

- The stress recorded with the second half bridge is almost 0 for the two considered variants $\left(\sigma_{2 \exp }=0,004\right.$ $\mathrm{MPa}$ - variant $1 ; \sigma_{2 \exp }=0,007 \mathrm{MPa}-$ variant 2);

- The main purpose of the study was to statically load the structure with a quite high value without reaching the plastic deformations

- The force has been gradually applied until we have reached a desired value; its value starts from zero at the begining of the experiment and returns to zero at the end of it (for a clear view, see fig. 8)

- The stress starts from zero at the begining of the experiment and returns to this value at the end of it (it means that we are in the elastic area of strains)

- The highest stress value is obtained for the variant 2: $\sigma_{1 \exp }=72,871 \mathrm{MPa}$

- In fig. 8 it is drawn the $\sigma_{1 \exp }$ variation depending on the value of force P; it starts and returns to zero at the begining and the end of the experiment; the area between the graphic lines represent the energy consumed by the structure during the experiment

- If we compare $\sigma_{1 \exp }$ with $\sigma_{2 \exp }$, the next results are obtained: $\sigma_{1 \exp }$ is 8567 times higher than $\sigma_{2 \exp }$ for the first variant and $\sigma_{1 \exp }$ is 10410 times higer than $\sigma_{2 \exp }$ for the second variant

Because the stress $\sigma_{2 \exp }$ values are very small compared to the $\sigma_{1 \exp }$ we shall consider their values neglibile

(equal to zero).

To demonstrate the accuracy of the presented device, we have compared the experimental results for the considered loading variants with the ones obtained from an analytical model, based on the classical force method and Mohr-Maxwell theorem, presented before in [Miritoiu et al., 2011][17]. The values are written in table 1 , where we have marked with $\sigma_{1 \text { the }}$ and $\sigma_{2 \text { the }}$ the stress values in the theoretical model corresponding to the places where we have the two half bridges from the strain gauges.

Table 1. The stress values for experimental and analytical methods

\begin{tabular}{|c|c|c|c|c|}
\hline \multicolumn{5}{|c|}{ Experimental Model (presented in this paper) } \\
\hline Stress type & $\sigma_{1 \exp }$ & $\sigma_{2 \exp }$ & $\sigma_{1 \exp }$ & $\sigma_{2 \exp }$ \\
\hline Loading variant & $1(\mathrm{P}=7,0155 \mathrm{kN}$ & $1(\mathrm{P}=7,0155 \mathrm{kN}$ & $2(\mathrm{~F}=5,0756 \mathrm{kN}$ & $2(\mathrm{~F}=5,0756 \mathrm{kN}$ \\
\hline $\begin{array}{c}\text { Stress Value } \\
{[\mathrm{MPa}]}\end{array}$ & 34,269 & $0,004 \approx 0$ & 72,871 & $0,007 \approx 0$ \\
\hline \multicolumn{5}{|c|}{ Analytical Model (from [Miritoiu et al., 2011][17]) } \\
\hline Stress type & $\sigma_{1 \text { the }}$ & $\sigma_{2 \text { the }}$ & $\sigma_{1 \text { the }}$ & $\sigma_{2 \text { the }}$ \\
\hline Loading variant & $1(\mathrm{P}=7,0155 \mathrm{kN}$ & $1(\mathrm{P}=7,0155 \mathrm{kN}$ & $2(\mathrm{~F}=5,0756 \mathrm{kN}$ & $2(\mathrm{~F}=5,0756 \mathrm{kN}$ \\
\hline $\begin{array}{c}\text { Stress Value } \\
{[\mathrm{MPa}]}\end{array}$ & 34,189 & 0 & 75,881 & 0 \\
\hline
\end{tabular}

I have determined the errors between the two models and I have written them in table 2.

Table.2.The errors values between the experimental and analytical methods

\begin{tabular}{|c|c|c|c|c|}
\hline \multicolumn{5}{|c|}{ Experimental Model / Analytical Model } \\
\hline Stress type & $\sigma_{1 \exp }$ & $\sigma_{2 \exp }$ & $\sigma_{1 \exp }$ & $\sigma_{2 \exp }$ \\
\hline Loading variant & $1(\mathrm{P}=7,0155 \mathrm{kN}$ & $1(\mathrm{P}=7,0155 \mathrm{kN}$ & $2(\mathrm{~F}=5,0756 \mathrm{kN}$ & $2(\mathrm{~F}=5,0756 \mathrm{kN}$ \\
\hline Error [\%] & 0,233 & 0 & 3,967 & 0 \\
\hline
\end{tabular}

From table 2 we can extract the following conclusions:

- Very small errors are obtained, under 4\%, accepted in practical engineering

- The device and method are accurate and can be succesfully applied when dealing with such problems

- The highest error is obtained for the second loading variant and it is 3,967\%

As a future research, I would consider the validation of this device with some results obtained by finite element models. 


\section{ACKNOWLEDGEMENT}

This work was partially supported by the strategic grant POSDRU/88/1.5/S/50783 (2009), co-financed by the European Social Fund - Investing in People, within the Sectoral Operational Programme Human Resources Development 2007-2013.

\section{REFERENCES}

[1] V. Roşca, et. al., Strength of Materials. Fundamental Testings (Universitaria, Craiova, 2007)

[2] A. Codatto, Method and device for bending elements such as pannels, metal sheet, plates or suchlike (Patent, Patent Number US 7.055.355 B2, http://ro.espacenet.com, 2006)

[3] G. Del Fabro, Bending device for a bending machine for oblong metal elements and relative bending method (Patent, International Patent Classification B21D 7/022, International Application Number PCT/EP2009/055421, http://ro.espacenet.com, 2009)

[4] H. Ernst and S. Manfred, Device for bending plates (Patent, International Patent Classification B21D1908, Published as EP1238723 (A3), http://ro.espacenet.com, 2002)

[5] B. Fishreder and T. Reiter, Bending press with a workpiece positioning device and an operating method (Patent, Classification: B21D43/00, Patent Number: WO2011AT00212 20110505, Published as: AT509857 (B1), http://ro.espacenet.com, 2012)

[6] T. Yogo, Bending Device (Patent Application, Publication Number: US2012/0016512, International Classification B25J 9/06, http://ro.espacenet.com, 2012)

[7] K. Shigeki, et. al., Device and method for bending metal tube (Patent, Patent Number: JP2009 0287404 20091218, Classification B25J 9/06, http://ro.espacenet.com, 2012)

[8] O. Yoshiaki, Device and method for bending metal bar material (Patent, Patent Number: B21D710, Classification: B21DT/10, http://ro.espacenet.com, 2011)

[9] H. Felix, et. al., Method and device for determining a bending angle of a rotor blade of a wind turbine (Patent, Patent Number: WO2011EP03293 20110702, Published as: DE 10201 0032120, http://ro.espacenet.com, 2012)

[10] W. Montero and R. Farag, Uncertainties Associated with strain-measuring systems using resistance strain gauges, The Journal of Strain Analysis for Engineering Design 40(7), 2005, 611-619

[11] A. Ajovalaist and B. Zucarello, Local reinforcement effect of a strain gauge installation on low modulus materials, The Journal of Strain Analysis for Engineering Design 40(7), 2005, 643-653

[12] M. Litos et. al., Simulation of strain gauge thermal effects during residual stress hole drilling measurements, The Journal of Strain Analysis for Engineering Design 40(7), 2005, 611-619

[13] M. Avalle and L. Goglio, Static Lateral Compression of aluminium tubes: strain gauge measurements and discussion of theoretical models, The Journal of Strain Analysis for Engineering Design 32(5), 1997, 335-343

[14] N. Faur, et. al., Stress-strain analysis for the wheel-railtrack assembly at urban passenger transport, International Conference Structural Integrity of Welded Structures ISCS 2007, Timisoara, Romania, 1-7, available at www.ndt.net, 2007

[15] I. Jonsson, et. al., Strain gauge measurement of wheel-rail interaction forces, The Journal of Strain Analysis for Engineering Design 32(3), 1997, 183-191

[16] T. Bandula-Heva and M. Dhanasekar, Determination of stress-strain characteristics of railhead steel using image analysis, World academy of Science, Engineering and Technology 60(335), 2011, 1884-1888

[17] C. M. Miriţoiu, et. al., An analytical model for strength calculus of hiperstatic structures, Journal of Engineering Studies and Research 7(3), 2011, 48-55 